\title{
Stability analysis of pressure and penetration rate in rotary drilling system
}

\author{
RHOUMA MLAYEH \\ Mathematics \& Computer Sciences Department \\ Carthage University - INSAT \\ LIM laboratory, Polytechnic School of Tunisia, BP 743, 2078 La Marsa, Tunisia \\ TUNISIA
}

\begin{abstract}
The purpose of this paper is to stabilize the annular pressure profile throughout the wellbore continuously while drilling. A new nonlinear dynamical system is developed and a controller is designed to stabilize the annular pressure and achieve asymptotic tracking by applying feedback control of the main pumps. Hence, the paper studies the control design for the well known Managed Pressure Drilling system (MPD). MPD provides a closed-loop drilling process in which pore pressure, formation fracture pressure, and bottom-hole pressure are balanced and managed at the surface. Although, responses must provide a solution for critical downhole pressures to preserve drilling efficiency and safety. Our MPD scheme is elaborated in reference to a nontrivial back-stepping control procedure and the effectiveness of the proposed control laws are shown by simulations.
\end{abstract}

Key-Words: - Drilling - Stability - Back-Stepping - Ordinary Differential Equation

Received: May 7, 2021. Revised: November 28, 2021. Accepted: December 21, 2021. Published: January 3, 2022.

\section{Introduction}

In recent century, the gas and oil industry has made great strides in developing drilling techniques and technologies that makes well construction a cost effective and safe enterprize. Also, a new techniques and approaches are developed in several research $[1,2,3,4,5,6,7,8,9,10,11,12,13]$. For the modeling, analysis and control of drilling systems, various works studies have investigated the stability properties of the drill string system. There exists three main type of vibrations: axial (bit-bouncing phenomenon), lateral (whirling phenomenon), and torsional (stick-slip oscillations). However, in the literature, many authors were interested in stabilizing the system with different techniques: Backstepping, Flatness, etc. Several authors have investigated the dynamics of oil well drilling, for example [6]. The energy function is proposed by Saldivar et al. [6] for the torsional model allows to find a control law that proves the energy dissipation during the drilling. During drilling operations, downhole cuttings need to be transported out of the bore hole. This is done by using a Managed Pressure Drilling (MPD) system. There exists several works were interested in stabilizing the MPD with different ways. An MPD is used to control the annular pressure profile throughout the well bore. The main objectives are to ascertain the downhole pressure environment limits and to manage the annular hydraulic pressure profile accordingly. The MPD is intended to suppress continuous influx of formation fluids to the surface. It is a new technology which has capability of mitigating drilling hazards, increasing production rates, and improving drilling performance. Consequently, the MPD will increase reserves by enabling drilling of areas that were previously economically unbribable. In the literature, different aspects of modeling for MPD have been proposed. Estimation and control design in MPD has been studied by several researchers so far [14]. Various challenges of modeling drilling systems for control and automation are discussed in [15].

In this work, we use the backstepping technique and Lyapunov theory to study our MPD. The backstepping technique is developed in 1990 by Petar V. Kokotovic et al. [16, 17] for analyzing the stability of the feedback controls of nonlinear dynamical systems. Then, it has becomes a useful tool in the feedback linearization of nonlinear systems of ODEs. In the Literature, various research are used backstepping method and Lyapunov theory to show the stability of nonlinear systems [ [16, 18]. Also, several results are invented in the stability studying of ODEs. In [19], the authors are used backstepping method, and Lyapunov theory to find the controller law. In [16], Kristick et al. are introduced a new method for nonlinear system with feedback control law. Roger et al. are provided the analysis of different techniques: two methods for find feedback control law are proposed and analyzed [20].

The main available variables in this paper are the injected pressure and the pump flow rate of water. Hence, our MPD study is different from the exist- 
ing approach in gas and oil industry. This can be explained by the fact that no choke pressure feedback in our drilling system. The most important challenge in these drilling operations is to control bottomhole pressure and penetration rate of the bit.

The paper is organized as follows: in Section 2, we present a hydraulic model (MPD) based on mass balanced and momentum balances that provides the governing equations for flow and pressure in the well in an MPD. We describe and we will formulate our system by a variable change. The main purpose for this section is to control the pump pressure at the bit, the penetration rate, and also the rotational drill string velocity. In section 2 , we illustrate the relevance and merits in numerical simulations. Some concluding and remarks are given in this last section.

\section{Modeling and stability analysis of an MPD}

\subsection{Model Description}

The Managed Pressure Drilling (MPD) system is used to control the pressure throughout the borehole in an oil well drilling. During drilling operation, a carefully designed fluid is pumped down from the mud pit through the drill string system, through the drill bit, up the annulus around the drill string, and back to the mud pit. The goal is not only to transport cuttings in the annulus, but also to manage the pressure in the well so that the unwanted inflow from the surrounding formation or well fracturing can be avoided. The

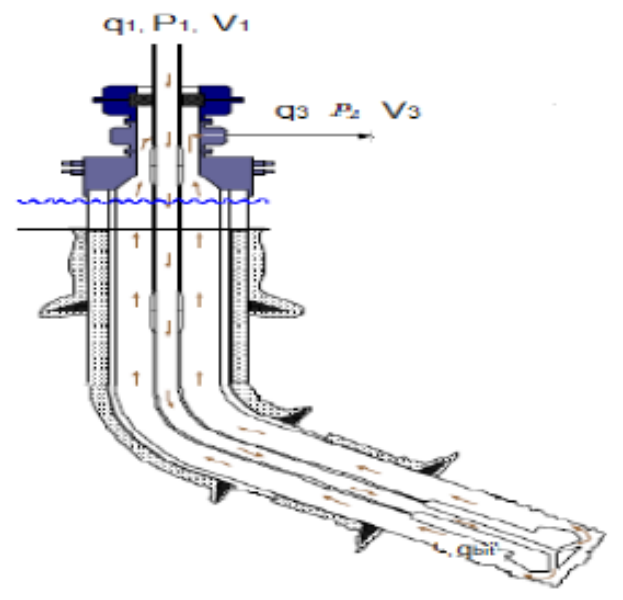

Figure 1: MPD in Rotary Drilling System.

hydraulic model of an MPD system (Fig. 1) derived from mass and momentum balances is described by the following system

$$
\begin{array}{cc}
M \dot{q}_{b i t}(t) & =P_{1}(t)-P_{2}(t)- \\
T\left(q_{b i t}\right)+ & g\left(\rho_{1} L-\rho_{3} y(t)\right) \\
=q_{1}(t)-q_{b i t}(t) & \\
\frac{V_{1}}{\beta_{1}}(t) & =u_{4} \\
\left(V_{0}+S y(t)\right) \dot{P}_{2}(t) & =\beta_{3}[q_{b i t}(t)+q_{2}(t, \Omega)-q_{3}(t)-\underbrace{\left.\frac{d V_{2}}{d t}\right]}_{S v(t)} \\
I \dot{\Omega} & =v(t) \\
\dot{y}(t) & =u_{2}(t) \\
\dot{v}(t) &
\end{array}
$$

where $L$ is the length of the well from mud pump, $y(t) \in[0, L]$ the spatial coordinate along the flow trajectory $(g$ gravity), $V_{2}=V_{0}+S y(t)$ is the crown volume, $v$ the penetration rate of the bit, $q_{1}$ the pump flow, $q_{2}$ the flow which describes the amount of flow through the surface, $S$ is the annular surface, $q_{3}$ is the flow out of the crown, $V_{1}$ is the volume of drill string, $\beta_{1}$ and $\beta_{3}$ are the effective bulk modulus, $q_{b i t}$ is the flow rate from the tool, $M$ is the integrated density per cross section, $P_{1}$ is the pump pressure, $P_{2}$ is the pressure when $y=L, I$ is the inertia of the drill string per unit length, $\rho_{1}$ is the density of the drilling mud in the drill string, $\rho_{3}$ is the annulus density, $\Omega$ is the rotational velocity of the drill string, $u_{4}$ is the control input from the torque applied to the drill string and $u_{2}$ is the control input from the penetration rate of the bit.

The total pressure drop due to the friction on the drill string and the annulus is represented by $T\left(q_{b i t}\right)$.

Assumption. The flow $q_{2}$ does not depend on the penetration rate, therefore the state $v$. Thus, it is initially considered that the torsion vibration phenomenon is not affected by the fluid injection.

For flow determination $q_{2}$ two cases occur.

\subsection{Case where the flow $q_{2} \triangleq q_{2}(t)$}

This case presents itself under a constant rotational speed $\Omega$ of the train-bit set. Thus, the flow $q_{2}$ (sol/ bit interaction) do not depend on the state of the system. Consequently, the system (1) - (6) is transformed to the following

$$
\begin{aligned}
M \dot{q}_{b i t}(t) & =P_{1}(t)-P_{2}(t)-T\left(q_{b i t}\right)+g\left(\rho_{1} L-\rho_{3} y(t)\right) \\
\frac{V_{1}}{\beta_{1}} \dot{P}_{1}(t) & =q_{1}(t)-q_{b i t}(t) \\
\left(V_{0}+S y(t)\right) \dot{P}_{2}(t) & =\beta_{3}[q_{b i t}(t)+q_{2}(t)-q_{3}(t)-\underbrace{\left.\frac{d V_{2}}{d t}\right]}_{S v(t)} \\
\dot{y}(t) & =v(t) \\
\dot{v}(t) & =u_{2}(t)
\end{aligned}
$$

Let us introduce $q_{1}=u_{1}$ as a control input in forces. We use the variable change $z(t)=\frac{1}{V_{0}+S y(t)}$, then we get the following system

$$
\begin{aligned}
\dot{q}_{b i t}(t) & =c_{1} P_{1}(t)-c_{1} P_{2}(t)-c_{1} T\left(q_{b i t}\right)+h(z) \\
\dot{P}_{1}(t) & =c u_{1}(t)-c q_{b i t}(t) \\
\dot{P}_{2}(t) & =R(t) z(t)-\beta_{3} z(t) S v(t) \\
\dot{z}(t) & =-S z^{2}(t) v(t) \\
\dot{v}(t) & =u_{2}(t)
\end{aligned}
$$


where $c=\frac{\beta_{1}}{V_{1}}, h(z)=c_{1} g\left(\rho_{1} L-\frac{\rho_{3}}{S}\left(\frac{1}{z(t)}-V_{0}\right)\right), c_{1}=\frac{1}{M}$, $R(t)=\beta_{3}\left[q_{b i t}(t)+q_{2}(t)-q_{3}(t)\right]$.

The equilibrium point of the system (17)-(11) is $\left(0,0,0, \frac{\rho_{3}}{\rho_{3} V_{0}+S \rho_{1} L}, 0\right)$.

The key contribution in this section, is the stability study of the ODE which describes the MPD under $\left(u_{1}, u_{2}\right)$ as control inputs from the injected fluid (the pump pressure and the penetration rate of the bit). Hence our goal is to control the pump pressure and the penetration rate.

Now, we study the result that leads to the control laws of the hydraulic system.

Theorem 1 Let consider the system (7)-(11). Choosing $A<0$ such that $\beta_{3} P_{2}(t) S z(t)+S z^{2}(t)\left(z(t)-\frac{\rho_{3}}{\rho_{3} V_{0}+S \rho_{1} L}\right)+A$ is never to zero. Then the both feedback control laws

$$
\begin{aligned}
u_{1}(t) & =-\frac{P_{1}-\Psi_{1}\left(q_{b i t}, P_{2}, z\right)}{c}+\frac{c-c_{1}}{c} q_{b i t}(t) \\
& +\frac{1}{c} \dot{\Psi}_{1}\left(q_{b i t}, P_{2}, z\right)
\end{aligned}
$$

and

$$
\begin{aligned}
u_{2}(t) & =S z^{2}(t)\left(z(t)-\frac{\rho_{3}}{\rho_{3} V_{0}+S \rho_{1} L}\right)-v(t)+\Psi_{2}\left(P_{2}, z\right) \\
& +\dot{\Psi}_{2}\left(P_{2}, z\right)+\beta_{3} S z(t) P_{2}(t)
\end{aligned}
$$

asymptotically stabilize the system (7)-(11) where

$$
\Psi_{1}\left(q_{b i t}, P_{2}, z\right)=P_{2}-\left(\frac{1}{c_{1}} q_{b i t}-T\left(q_{b i t}\right)\right)-\frac{h(z)}{c_{1}}
$$

and

$$
\Psi_{2}\left(P_{2}, z\right)=\frac{P_{2}^{2}(t)+\left(z(t)-\frac{\rho_{3}}{\rho_{3} V_{0}+S \rho_{1} L}\right)^{2}+\left(\frac{P_{2}(t)}{2}+R(t) z(t)\right)^{2}}{\beta_{3} P_{2}(t) S z(t)+S z^{2}(t)\left(z(t)-\frac{\rho_{3}}{\rho_{3} V_{0}+S \rho_{1} L}\right)+A}
$$

in which

$$
\Psi_{2}\left(0, \frac{\rho_{3}}{\rho_{3} V_{0}+S \rho_{1} L}\right)=0, \quad \Psi_{1}\left(0,0, \frac{\rho_{3}}{\rho_{3} V_{0}+S \rho_{1} L}\right)=0
$$

In order to prove this theorem, we use the backstepping technique and the Lyapunov theory.

Proof 1 First, we consider the following sub-system

$$
\dot{q}_{b i t}(t)=c_{1} P_{1}(t)-c_{1} P_{2}(t)-c_{1} T\left(q_{b i t}\right)+h(z)
$$

and introduce a virtual feedback control law which satisfies

$$
\Psi_{1}\left(q_{b i t}, P_{2}, z\right)=P_{2}-\left(\frac{1}{c_{1}} q_{b i t}-T\left(q_{b i t}\right)\right)-\frac{h(z)}{c_{1}}
$$

in which $\Psi_{1}\left(0,0, \frac{\rho_{3}}{\rho_{3} V_{0}+S \rho_{1} L}\right)=0$. Then we obtain

$$
\dot{q}_{b i t}(t)=-q_{b i t}
$$

We introduce the following Lyapunov function $L_{1}(t)=\frac{1}{2} q_{b i t}^{2}$, then $\dot{L}_{1}(t)=-2 L_{1}(t)$. This proves that (7) is asymptotically stable at the equilibrium $q_{\text {bit }}=0$.

Second, let consider the virtual state variable

$$
\zeta_{1}=P_{1}-\Psi_{1}\left(q_{b i t}, P_{2}, z\right)
$$

Then we obtain

$$
\begin{aligned}
\dot{q}_{b i t}(t) & =c_{1} \zeta_{1}(t)-q_{b i t}(t) \\
\dot{\zeta}_{1}(t) & =-\dot{\Psi}_{1}\left(q_{b i t}, P_{2}, z\right)+c u_{1}(t)-c q_{b i t}(t)
\end{aligned}
$$

We choose the following Lyapunov function

$$
L_{2}(t)=L_{1}(t)+\frac{1}{2} \zeta_{1}^{2}=\frac{1}{2}\left(q_{b i t}^{2}+\zeta_{1}^{2}\right)
$$

The time derivative of $L_{2}$ is given by

$$
\begin{aligned}
\dot{L}_{2} & =\dot{q}_{b i t} q_{b i t}+\dot{\zeta}_{1} \zeta_{1} \\
& =q_{b i t}\left(c_{1} \zeta_{1}-q_{b i t}\right)+\zeta_{1}\left(-\dot{\Psi}_{1}\left(q_{b i t}, P_{2}, z\right)+c u_{1}(t)\right. \\
& \left.-c q_{b i t}(t)\right) \\
& =-q_{b i t}^{2}-\zeta_{1}^{2}+\zeta_{1}\left(\zeta_{1}+\left(c_{1}-c\right) q_{b i t}-\dot{\Psi}_{1}\left(q_{b i t}, P_{2}, z\right)\right. \\
& \left.+c u_{1}(t)\right)
\end{aligned}
$$

Here, we select the actual feedback control law

$$
u_{1}(t)=-\frac{\zeta_{1}}{c}+\frac{c-c_{1}}{c} q_{b i t}(t)+\frac{1}{c} \dot{\Psi}_{1}\left(q_{b i t}, P_{2}, z\right)
$$

This proves that $\dot{L}_{2}(t)=-2 L_{2}(t)$. Then the system (7)-(8) is asymptotically stable at the equilibrium $\left(q_{b i t}, P_{1}\right)=(0,0)$. Now, we consider the following sub-system

$$
\begin{aligned}
\dot{P}_{3}(t) & =R(t) z(t)-\beta_{3} z(t) S v(t) \\
\dot{z}(t) & =-S z^{2}(t) v(t) \\
\dot{v}(t) & =u_{2}(t)
\end{aligned}
$$

As before, let consider

$$
\begin{aligned}
\dot{P}_{3}(t) & =R(t) z(t)-\beta_{3} S z(t) v(t) \\
\dot{z} & =-S v(t) z^{2}(t)
\end{aligned}
$$

where $R(t)=\beta_{3}\left(q_{b i t}(t)+q_{2}(t)-q_{3}(t)\right)$, and introduce a virtual feedback control law which might $\Psi_{2}\left(P_{2}, z\right)=$ $\frac{P_{2}^{2}(t)+\left(z(t)-\frac{\rho_{3}}{\rho_{3} V_{0}+S \rho_{1} L}\right)^{2}+\left(\frac{1}{2} P_{2}(t)+R(t) z(t)\right)^{2}}{\beta_{3} P_{2}(t) S z(t)+S z^{2}(t)\left(z(t)-\frac{\rho_{3}}{\rho_{3} V_{0}+S \rho_{1} L}\right)+A}$

in which $\Psi_{2}\left(0, \frac{\rho_{3}}{\rho_{3} V_{0}+S \rho_{1} L}\right) \stackrel{\rho_{3} v_{0}+S \rho_{1}}{=} 0$.

Then we obtain the following system

$$
\begin{aligned}
\dot{P}_{3}(t) & =R(t) z(t)-\beta_{3} z(t) S \Psi_{2}\left(P_{2}, z\right) \\
\dot{z} & =-S z^{2}(t) \Psi_{2}\left(P_{2}, z\right)
\end{aligned}
$$

We use the following Lyapunov function

$$
L_{3}(t)=\frac{1}{2}\left(P_{2}^{2}+\left(z-\frac{\rho_{3}}{\rho_{3} V_{0}+S \rho_{1} L}\right)^{2}\right)
$$

The time derivative of $L_{3}$ is given by

$$
\begin{aligned}
\dot{L}_{3}(t) & =\dot{P}_{2} P_{2}+\dot{z}\left(z-\frac{\rho_{3}}{\rho_{3} V_{0}+S \rho_{1} L}\right) \\
& =R(t) z(t) P_{2}(t)-\beta_{3} S z(t) P_{2}(t) \Psi_{2}\left(P_{2}, z\right) \\
& -S z^{2}(t)\left(z-\frac{\rho_{3}}{\rho_{3} V_{0}+S \rho_{1} L}\right) \Psi_{2}\left(P_{2}, z\right) \\
& \leq R(t) z(t) P_{2}(t)-\left[P_{2}^{2}(t)+\left(z-\frac{\rho_{3}}{\rho_{3} V_{0}+S \rho_{1} L}\right)^{2}\right. \\
& \left.+\left(\frac{1}{2} P_{2}(t)+R(t) z(t)\right)^{2}\right] \\
& \leq-P_{2}^{2}(t)-\left(z-\frac{\rho_{3}}{\rho_{3} V_{0}+S \rho_{1} L}\right)^{2} \\
& \leq-2 L_{3}(t)
\end{aligned}
$$


This proves that the system (12)-(13) is asymptotically stable at the equilibrium $(P, z)=\left(0, \frac{\rho_{3}}{\rho_{3} V_{0}+S \rho_{1} L}\right)$.

Second, we use the following virtual state variable, $\zeta_{2}(t)=$ $v(t)-\Psi_{2}(P, z)$ to transform the system (12)-(14) in this form

$$
\begin{aligned}
\dot{P}_{3}(t) & =R(t) z(t)-\beta_{3} S z(t) \zeta_{2}(t)-\beta_{3} z(t) S \Psi_{2}\left(P_{2}, z\right) \\
\dot{z}(t) & =-S z^{2}(t) \zeta_{2}(t)-S z^{2}(t) \Psi_{2}\left(P_{2}, z\right) \\
\dot{\zeta_{2}}(t) & =u_{2}(t)-\dot{\Psi}_{2}\left(P_{2}, z\right)
\end{aligned}
$$

We know that the first sub-system $(15)-(16)$ is asymptotically stable at the equilibrium $(P, z)=\left(0, \frac{\rho_{3}}{\rho_{3} V_{0}+S \rho_{1} L}\right)$. Here, we use the following Lyapunov function

$$
L_{4}(t)=L_{3}(t)+\frac{1}{2} \zeta_{2}^{2}
$$

We differentiate $L_{4}$ with respect to time, we get

$$
\begin{aligned}
\dot{L}_{4}(t) & =\dot{P}_{2}(t) P_{2}(t)+\dot{z}(t)\left(z(t)-\frac{\rho_{3}}{\rho_{3} V_{0}+S \rho_{1} L}\right)+\dot{\zeta}_{2}(t) \zeta_{2}(t) \\
& \leq-2 L_{3}+\dot{\zeta}_{2}(t) \zeta_{2}(t)-\beta_{3} S z(t) \zeta_{2}(t) P_{2}(t) \\
& -S z^{2}(t)\left(z(t)-\frac{\rho_{3}}{\rho_{3} V_{0}+S \rho_{1} L}\right) \zeta_{2}(t) \\
& \leq-2 L_{3}(t)-\zeta_{2}^{2}(t)+\left(\zeta_{2}(t)+u_{2}(t)-\dot{\Psi}_{2}\left(P_{2}, z\right)\right) \zeta_{2}(t) \\
& -\beta_{3} S z(t) \zeta_{2}(t) P_{2}(t)-S z^{2}(t)\left(z(t)-\frac{\rho_{3}}{\rho_{3} V_{0}+S \rho_{1} L}\right) \zeta_{2}(t) \\
& \leq-2 L_{4}(t)+\left(\zeta_{2}(t)+u_{2}(t)-\dot{\Psi}_{2}\left(P_{2}, z\right)\right. \\
& \left.-\beta_{3} S z(t) P_{2}(t)-S z^{2}(t)\left(z(t)-\frac{\rho_{3}}{\rho_{3} V_{0}+S \rho_{1} L}\right)\right) \zeta_{2}(t)
\end{aligned}
$$

Hence, we select the actual control law given by

$$
\begin{aligned}
u_{2}(t) & =S z^{2}(t)\left(z(t)-\frac{\rho_{3}}{\rho_{3} V_{0}+S \rho_{1} L}\right)-\zeta_{2}(t) \\
& +\dot{\Psi}_{2}\left(P_{2}, z\right)+\beta_{3} S z(t) P_{2}(t)
\end{aligned}
$$

Then $\dot{L}_{4}(t) \leq-2 L_{4}(t)$.

Consequently, let introduce the following Lyapunov function

$$
\begin{aligned}
V(t) & =\frac{1}{2}\left(q_{b i t}^{2}+\left(P_{1}-\Psi_{1}\left(q_{b i t}, P_{2}, z\right)\right)^{2}\right) \\
& +\frac{1}{2}\left(P_{3}^{2}(t)+\left(z(t)-\frac{\rho_{3}}{\rho_{3} V_{0}+S \rho_{1} L}\right)^{2}\right) \\
& +\frac{1}{2}\left(v(t)-\Psi_{2}\left(P_{2}, z\right)\right)^{2}
\end{aligned}
$$

Then

$$
\dot{V}=\dot{L}_{2}+\dot{L}_{4} \leq-2\left(L_{2}+L_{4}\right) \leq-2 V
$$

This conclude that the system (7)-(11) is asymptotically stable at the equilibrium.

Remark 1 The torsional vibration phenomenon is considered to be independent of the injection system, this implies that the penetration rate of the bit is not impacted by the pressure of the injected fluid. Indeed, in the contrary case, we will have to deal with a model coupling the dynamics of torsion as well as the fluid behavior injected by the hydraulic system to the surface.

\subsection{Case where the flow $q_{2} \triangleq q_{2}(t, \Omega)$}

This case occurs at a non constant rotational velocity $\Omega$ of the drill string. Then, the flow $q_{2}$ (bit / rock interaction) depends on the state of the system. So, we can express:

$$
\begin{aligned}
q_{2}(t, \Omega) & =\rho_{3} \operatorname{Sr}(\Omega(t)+d(t)) \\
I \dot{\Omega} & =u_{1}(t)
\end{aligned}
$$

where $d(t)$ is the disturbance, $I$ the inertia of the drill string per unit length, $r$ the annular radius, $u_{4}$ the torque applied to the drill string. Our main is to control the pressure at the bit, the penetration rate, and also the rotational drill string velocity.

Consequently, we consider the following model

$$
\begin{aligned}
\dot{q}_{b i t}(t) & =c_{1} P_{1}(t)-c_{1} P_{2}(t)-c_{1} T\left(q_{b i t}\right)+h(z) \\
\dot{P}_{1}(t) & =c u_{1}(t)-c q_{b i t}(t) \\
\dot{P}_{3}(t) & =R(t) z(t)+\beta_{3} \rho_{3} S r(w(t)+d(t)) z(t) \\
& -\beta_{3} z(t) S v(t) \\
\dot{\Omega} & =\frac{1}{I} u_{4}(t) \\
\dot{z}(t) & =-S z^{2}(t) v(t) \\
\dot{v}(t) & =u_{3}(t)
\end{aligned}
$$

where $R(t)=\beta_{3}\left[q_{b i t}-q_{3}\right], u_{1}$ is considered an input control, $u_{4}$ is the torque applied to the drill string which control the amount of flow through a surface (tool/ground) at the bottomhole and $u_{3}$ is the penetration rate.

Theorem 2 The three feedback control laws

$$
\begin{aligned}
u_{1}(t) & =-\frac{P_{1}-\Psi_{1}\left(q_{b i t}, P_{2}, z\right)}{c}+\frac{c-c_{1}}{c} q_{b i t}(t)+\frac{1}{c} \dot{\Psi}_{1}\left(q_{b i t}, P_{2}, z\right) \\
u_{3} & =-v+\Psi_{4}+S z^{2}\left(z-\frac{\rho_{3}}{\rho_{3} V_{0}+\rho_{1} S L}\right)+\dot{\Psi}_{4} \\
u_{4}(t) & =I\left(-\rho_{3} S r \beta_{3} P_{2}(t) z(t)+\dot{\Psi}_{3}-\Omega+\Psi_{3}\right)
\end{aligned}
$$

asymptotically stabilize the system (17)-(22) at the equilibrium $\left(q_{b i t}, P_{1}, P_{2}, \Omega, z, v\right)=\left(0,0,0,0, \frac{\rho_{3}}{\rho_{3} V_{0}+S \rho_{1} L}, 0\right)$ where

$$
\begin{aligned}
\Psi_{1}\left(q_{b i t}, P_{2}, z\right) & =P_{2}-\left(\frac{1}{c_{1}} q_{b i t}-T\left(q_{b i t}\right)\right)-\frac{h(z)}{c_{1}} \\
\Psi_{4}(z) & =\frac{z-\frac{\rho_{3}}{\rho_{3} V_{0}+\rho_{1} S L}}{S z^{2}(t)}
\end{aligned}
$$

$\Psi_{3}\left(P_{2}, z, v\right)=\frac{-P_{2}-R(t) z(t)-\rho_{3} S r \beta_{3} d(t) z(t)+\beta_{3} S z(t) v(t)}{\rho_{3} S r \beta_{3} z(t)}$ in which $\Psi_{1}\left(0,0, \frac{\rho_{3}}{\rho_{3} V_{0}+S \rho_{1} L}\right) \stackrel{\rho_{3} S r \rho_{3} z(t)}{=} \Psi_{3}\left(0, \frac{\rho_{3}}{\rho_{3} V_{0}+S \rho_{1} L}, 0\right)=0$ and $0=\Psi_{4}\left(\frac{\rho_{3}}{\rho_{3} V_{0}+S \rho_{1} L}\right)$.

Proof 2 We recall that the system $(\sqrt{17})-(\sqrt{18})$ is asymptotically stable at the equilibrium $\left(q_{b i t}, P_{1}\right)=(0,0)$ (see proof of Theorem [1), consequently the system (17)-(18) is asymptotically stable at the same equilibrium.

Now, let consider the following sub-system

$$
\begin{aligned}
\dot{P}_{3}(t) & =R(t) z(t)+\beta_{3} \rho_{3} S r(\Omega(t)+d(t)) z(t)-\beta_{3} z(t) S v(t) \\
\dot{\Omega} & =\frac{1}{I} u_{4}(t)
\end{aligned}
$$

We introduce the virtual control law $\Psi_{3}\left(P_{2}, z, v\right)=\frac{-P_{2}-R(t) z(t)-\rho_{3} S r \beta_{3} d(t) z(t)+\beta_{3} S z(t) v(t)}{\rho_{3} S r \beta_{3} z(t)}$. We 
propose $L_{5}(t)=\frac{1}{2} P_{2}^{2}$, then, we get $\dot{L_{5}}(t) \leq-P_{2}^{2}$.

Let $\xi_{3}(t)=\Omega(t)-\Psi_{3}$ the virtual state variable, then we obtain the following sub-system

$$
\begin{aligned}
\dot{P}_{3}(t) & =R(t) z(t)+\beta_{3} \rho_{3} \operatorname{Sr}\left(\xi_{3}(t)+\Psi_{3}+d(t)\right) z(t) \\
& -\beta_{3} z(t) S v(t) \\
\dot{\xi}_{3} & =-\dot{\Psi}_{3}+\frac{1}{I} u_{4}(t)
\end{aligned}
$$

Here, we introduce the following Lyapunov function

$$
L_{6}(t)=\frac{1}{2}\left(P_{2}^{2}(t)+\xi_{3}^{2}(t)\right)
$$

The time derivative of $L_{6}$ is given by

$$
\begin{aligned}
\dot{L}_{6}(t) & =\dot{P}_{3} P_{2}+\dot{\xi}_{3} \xi_{3} \\
& =P_{2}\left(R(t) z(t)+\beta_{3} \rho_{3} S r z(t) \Psi_{3}+\beta_{3} \rho_{3} \operatorname{Srd}(t) z(t)\right. \\
& \left.-\beta_{3} S z(t) v(t)\right)+\xi_{3}(t)\left(P_{2}(t) \rho_{3} S r \beta_{3} z(t)\right. \\
& \left.-\dot{\Psi}_{3}+\frac{1}{I} u_{4}(t)\right)
\end{aligned}
$$

We select the actual control law

$$
u_{4}(t)=I\left(-\rho_{3} S r \beta_{3} P_{2}(t) z(t)+\dot{\Psi}_{3}-\xi_{3}(t)\right)
$$

Then $\dot{L}_{6}(t) \leq-2 L_{6}(t)$.

Finally, let consider the last sub-system

$$
\begin{aligned}
\dot{z}(t) & =-S z^{2}(t) v(t) \\
\dot{v}(t) & =u_{3}(t)
\end{aligned}
$$

We consider $\Psi_{4}=\frac{z-\frac{\rho_{3}}{\rho_{3} V_{0}+\rho_{1} S L}}{s z^{2}(t)}$ as the virtual control law and the lyapunov function $L_{7}(t)=\frac{1}{2}\left(z-\frac{\rho_{3}}{\rho_{3} V_{0}+\rho_{1} S L}\right)^{2}$, we obtain $\dot{L}_{7}(t) \leq-2 L_{7}$. Let $\xi_{4}=v-\Psi_{4}$ the virtual state variable. Then we get the following sub-system

$$
\begin{aligned}
\dot{z}(t) & =-S z^{2}(t) \xi_{4}(t)-S z^{2}(t) \Psi_{4} \\
\dot{\xi}_{4}(t) & =-\dot{\Psi}_{4}+u_{3}(t)
\end{aligned}
$$

We introduce the following Lyapunov function

$L_{8}(t)=L_{7}(t)+\frac{1}{2} \xi_{4}^{2}(t)$. Differentiating $L_{8}$ with respect to time, we find

$$
\begin{aligned}
\dot{L}_{8}(t) & =\dot{L}_{7}(t)+\xi(t) \dot{\xi}(t) \\
& \leq-2 \dot{L}_{7}(t)+\xi_{4}\left(-S z^{2}\left(z-\frac{\rho_{3}}{\rho_{3} V_{0}+\rho_{1} S L}\right)\right. \\
& \left.-\dot{\Psi}_{4}+u_{3}(t)\right) \\
& \leq-2 \dot{L}_{8}(t)+\xi_{4}\left(\xi_{4}-S z^{2}\left(z-\frac{\rho_{3}}{\rho_{3} V_{0}+\rho_{1} S L}\right)\right. \\
& \left.-\dot{\Psi}_{4}+u_{3}(t)\right)
\end{aligned}
$$

Then selecting the actual control law $u_{3}$

$$
u_{3}=-\xi_{4}+S z^{2}\left(z-\frac{\rho_{3}}{\rho_{3} V_{0}+\rho_{1} S L}\right)+\dot{\Psi}_{4}
$$

Hence, let introduce the following Lyapunov function

$$
\begin{aligned}
\mathfrak{L} & =\frac{1}{2}\left[q_{b i t}^{2}+\left(P_{1}-\Psi_{1}\right)^{2}+P_{2}^{2}+\left(\Omega-\Psi_{3}\right)^{2}\right. \\
& \left.+\left(z-\frac{\rho_{3}}{\rho_{3} V_{0}+\rho_{1} S L}\right)^{2}+\left(v-\Psi_{4}\right)^{2}\right]
\end{aligned}
$$

Then

$$
\begin{aligned}
\dot{\mathfrak{L}} & =\dot{L}_{2}+\dot{L}_{6}+\dot{L}_{8} \\
& \leq-2\left(L_{2}+L_{6}+L_{8}\right) \leq-2 \mathfrak{L}
\end{aligned}
$$

Finally, we conclude that the overall system (17)-(22) is asymptotically stable.

\section{Simulation}

Drilling is an essential part of the oil industry and penetration rate must be enhanced to ensure speedy completion of drilling processs. Torque on bit, pressure, rotary speed, weight on bit, drill bit type, formation characteristics and mud properties are the basic factors that affect the penetration rate of a bit. The focus of this work is the stability of the MPD system under the control of fluid and penetration rate of the bit. Hence, in this section, we test the effectiveness of the control laws found for the stability of the MPD. As expected by Theorems 1 and 2, the proposed controller stabilizes all the drilling variables including the downhole pressure in the well. The following physical parameters are used in simulation [21, 4, 14]:

\begin{tabular}{ll} 
Variable & Value \\
\hline$L$ & $2000 \mathrm{~m}$ \\
$I$ & $0.095 \mathrm{~kg} \cdot \mathrm{m}$ \\
$\rho_{1}=\rho_{3}$ & $1250 \mathrm{~kg} \cdot \mathrm{m}^{-3}$ \\
$M$ & $8300 \mathrm{~kg} \cdot \mathrm{m}^{-4}$ \\
$\beta_{1}=\beta_{3}$ & $24750 \mathrm{bar}$ \\
$V_{0}$ & $110 \mathrm{~m}^{3}$ \\
$g$ & $9.81 \mathrm{~ms}^{-2}$ \\
$S$ & $\pi \times(0.25)^{2} \mathrm{~m}^{2}$ \\
$c_{d}$ & 0.61 \\
$T_{a}$ & $0.003 .10^{6} \frac{{\mathrm{bar} . \mathrm{s}^{2}}_{\mathrm{m}^{6}}}{}$
\end{tabular}

Table 1: Different physical parameters

The flow rate due to the mud leaving through the open annulus is given by [22],

$$
q_{3}=c_{d} S \sqrt{\frac{2}{\rho_{3}}\left(P_{d h}-P_{2}+\rho_{3} \frac{g}{S}\left(\frac{1}{z}-V_{0}\right)\right)}
$$

The displacement of the bit, and the bit characteristics permit to construct the bottomhole pressure

$$
P_{d h}=P_{2}-\frac{\rho_{3} g}{S}\left(\frac{1}{z}-V_{0}\right)+T_{a} q_{b i t}^{2}
$$

with $T_{a}$ denotes the friction factor in the annulus. The stabilizing controller results to MPD are presented in figures (Fig. 27). Clearly, all the simulations imply an adequate convergence of the system variables to their expected values. For example, one notes that $y$ converges to $\frac{\rho_{1} L}{\rho_{3}}=2000 . m$ (Fig. 2). By applying the control laws (Fig. 77-8), the equilibrium is reached asymptotically stable (Fig. 3-6). Consequently, under the proposed control input, the hydraulic vibration system avoids an excessive increase (saturation) in pressures. 


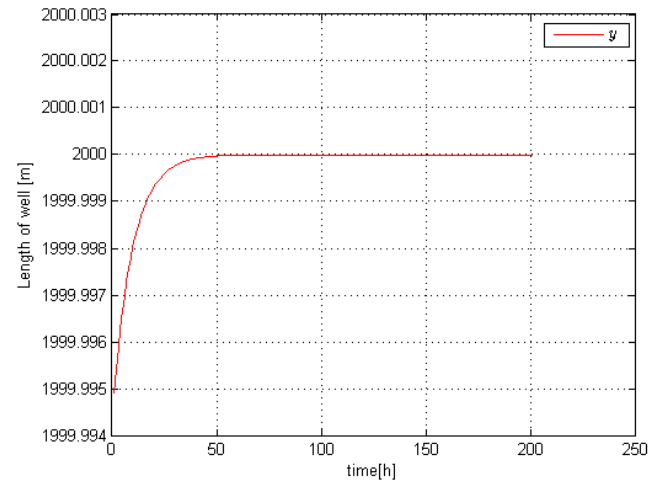

Figure 2: Stabilization of the state $y$

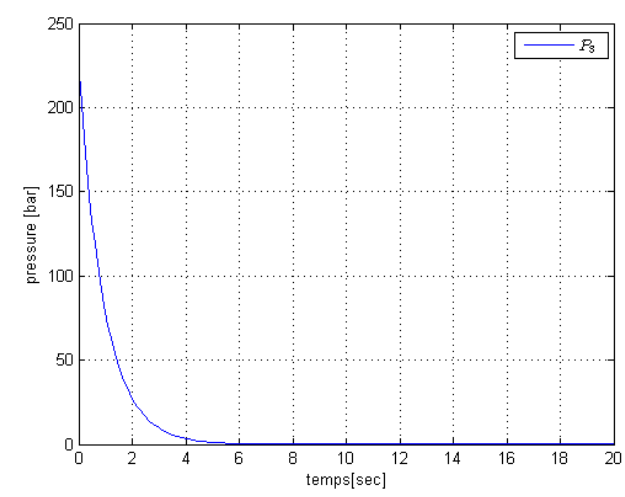

Figure 3: Stabilization of the pressure $P_{2}$

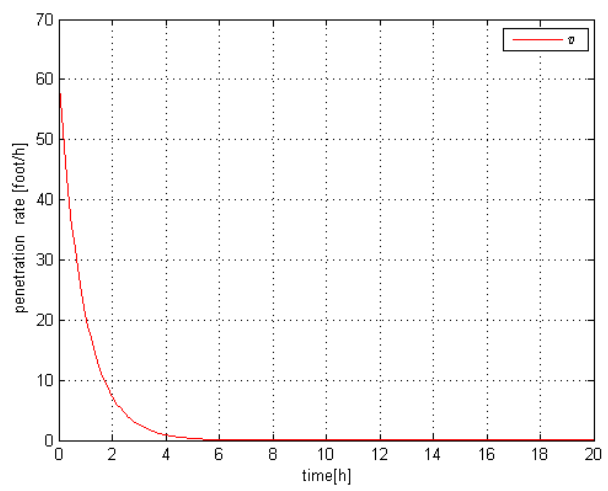

Figure 4: Stabilization of the penetration rate of the bit $v$

\section{Conclusion}

In this work, a dynamic model based on mass and momentum balances for MPD which describes by five and six ODE is proposed. The most important task in this paper is to control the pressure at the bit, the penetration rate, and bottom-hole pressure during drilling operations. The proposed controller laws asymptotically stabilizes all the drilling variables including the downhole pressure in the well. Future work should focus on the interaction between MPD system and torsional vibrations.

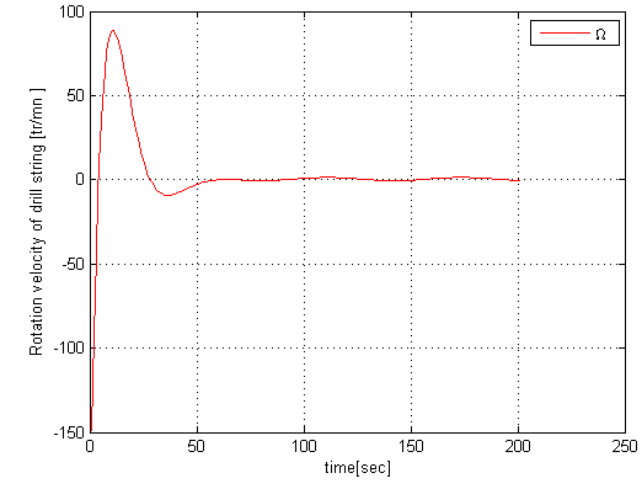

Figure 5: Stabilization of the rotation velocity of the drill string $\Omega$

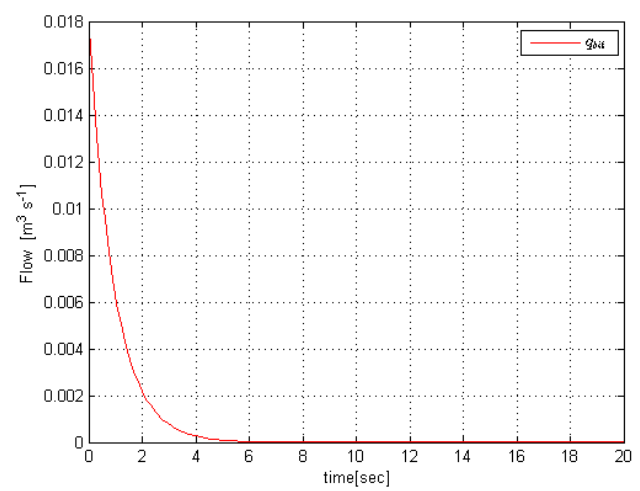

Figure 6: Stabilization of the the flow rate from the tool $q_{b i t}$

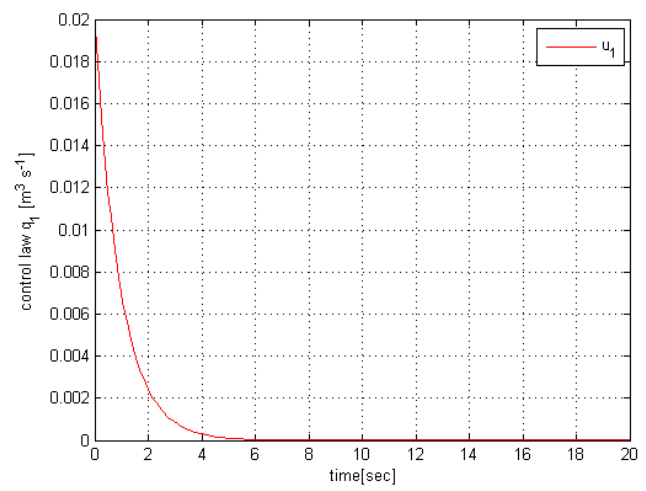

Figure 7: Stabilization of the control law $u_{1}$

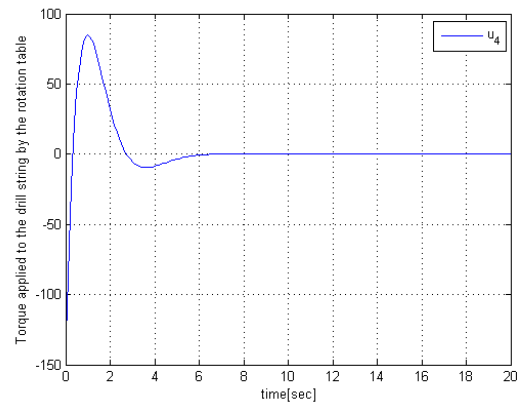

Figure 8: Stabilization of the control law $\mathrm{u}_{4}$ 


\section{References:}

[1]. S. Toumi and L. Beji and R. Mlayeh, Torsional Vibration Suppression with Boundary Impulsive Conditions in Rotary Drilling System, IIEEE 58th Conference on Decision and Control (CDC 2019), 2019, pp. 9951000.

[2] B. Saldivar and S. Mondié and S-I. N and H. Mounier and I. Boussaada, A control oriented guided tour in oilwell drilling vibration modeling, Annual Reviews in Control, 2016, pp. 100-113.

[3] E. Kreuzer and L. Krumm and M-A. Pick and E. Solowjow and M. Steidl, Active vibration isolation via decomposition of traveling waves, 11th International Conference on Vibration Problems, Lisbon, Portugal (9-12 September 2013), 2013.

[4] S. Toumi and L. Beji and R. Mlayeh and A. Abichou, Stability analysis of coupled torsional vibration and pressure in oilwell drillstring system, International Journal of Control (2017), Vol.91, No.1, 2017, pp. 241-252.

[5] X. Liu and N. Vlajic and X. Long and G. Meng, and B. Balachandran, "Coupled Axial-Torsional Dynamics in Rotary Drilling with State Dependent Delay: Stability and Control, Nonlinear Dynamics, Vol.78, No.3, 2014, pp. 1891-1906.

[6] B. Saldivar and S. Mondie and J. J. Loiseau and V. Rasvan, Stick- Slip Oscillations in Oillwell Drillstrings: Distributed Parameter and Neutral Type Retarded Model Approaches, Proceedings of the 18th IFAC World Congress, Vol.44, No.1, 2011, pp. 284-289.

[7] S. Toumi and L. Beji and R. Mlayeh and A. Abichou, Boundary Observer Design for Hyperbolic PDE in rotary drilling systems, IEEE 55th Conference on Decision and Control (CDC), Las Vegas, USA, 2016, pp. 2128-2133.

[8] Sicco Dwars, Shell, Recent Advances in Soft Torque Rotary Systems, Society of Petroleum Engineers, 2015, pp. 1-12.

[9] S. Toumi and R. Mlayeh and L. Beji and A. Abichou, Stability analysis of oilwell drilling torsional vibrations, Control and Automation (MED), 24th Mediterranean Conference, 2016, pp. 677-682.

[10] J.J. BAILEY and I.FINNIE, An analytical study of drillstring vibration, Journal of Engineering for Industry, Vol.82, No.2, 1960, pp. 122-127

[11] Y. N. Stamnes, Thesis for the degree of Philosophiae Doctor, NTNU Norwegian Universty Science of Technology, Trondheim (2011), 2011.

[12] E. Navarro-López and R. Suárez, Practical approach to modelling and controlling stick-slip oscillations in oilwell drillstrings, Control Applications. Proceedings of the 2004 IEEE International Conference, Vol.2, 2004, pp. 1454-1460.

[13] F. White, Fluid Mechanics, New York: McGraw-Hill (2008), 2008.

[14] G.O. Kaasa and . N. Stamnes and O. M. Aamo and and L. S. Imsland, Simplified hydraulics model used for intelligent estimation of downhole pressure for a managedpressure-drilling control system, SPE Drilling and Completion, Vol.27, No.1, 2012, pp. 127-138.
[15] G. C. Downton, Challenges of modeling drilling systems for the purposes of automation and control, IFAC Workshop on Automatic Control in Offshore Oil and Gas Production, Vol.45, No.8, 2012, pp. 201-210.

[16] M. Krstic and I. Kanellakopoulos and P. Kokotovic, Nonlinear and Adaptive Control Design, Wiley-Interscience, 1995.

[17] R. Lozano and B. Brogliato, Adaptive control of robot manipulators with flexible joints, IEEE, Vol.37, No.2, 1992, pp. 174-181.

[18] H. J. Marquez, Nonlinear Control Systems, Analysis and Design, IEEE TRANSACTIONS ON AUTOMATIC CONTROL, Vol.49, No.7, 2004, pp. 1225-1226.

[19] M.T.Alrifai; J.H.Chow and D.A.Torrey, A Backstepping Nonlinear Control Approach to Switched Reluctance Motors, Proceedings of the 37th IEEE Conference on Decision \& Control Tampa, Florida USA, 1998.

[20] R. Skjetne and T. I. Fossen, On Integral Control in Backstepping Analysis of Different Techniques, Proceeding of the 2004 Americain Control Conference Boston, Massachusettes, 2004, pp. 1899-1904.

[21] S. Toumi and L. Beji and R. Mlayeh and A. Abichou, Stabilization of torsional vibration in oilwell drillstring system, Eurpean Journal of Control (2017), Vol.35, 2017, pp. 19-27.

[22] S. Toumi and L. Beji and R. Mlayeh and A. Abichou, Stabilization of stick-slip oscillations Integrating fluid injection in oilwell drillstring system, European Control Conference, 2016, pp. 352-357.

\section{Creative Commons Attribution License 4.0 (Attribution 4.0 International, CC BY 4.0)}

This article is published under the terms of the Creative Commons Attribution License 4.0 https://creativecommons.org/licenses/by/4.0/deed.en US 